\title{
Laboratory Testing of Self-Healing Polymer Modified Asphalt Mixtures Containing Recycled Asphalt Materials (RAP/RAS)
}

\author{
Sharareh Shirzad ${ }^{1}$, Marwa M. Hassan ${ }^{1, *}$, Max A. Aguirre ${ }^{1}$, Samuel Cooper, Jr., ${ }^{2}$ Louay N. Mohammad ${ }^{3}$, and Ioan I. \\ Negulescu ${ }^{4}$ \\ ${ }^{1}$ Department of Construction Management, Louisiana State University, Baton Rouge, LA 70803 \\ ${ }^{2}$ Director, Louisiana Transportation Research Center, Baton Rouge, LA 70808 \\ ${ }^{3}$ Department of Civil and Environmental Engineering, Louisiana State University, Baton Rouge, LA 70803 \\ ${ }^{4}$ Department of Chemistry, Louisiana State University, Baton Rouge, LA 70803
}

\begin{abstract}
The objective of this study was to evaluate the efficiency of an innovative light-induced selfhealing polymers in enhancing the durability of asphalt mixtures and improving its self-healing properties. Mixtures were prepared using two different binders, with and without recycled materials, and self-healing polymer. Results showed that the addition of recycled asphalt material to mixtures prepared with an unmodified binder negatively affected the healing recovery at room temperature. Furthermore, Self-healing properties of the mixtures were improved by increasing the healing temperature. The addition of $5 \%$ selfhealing polymer to the control mixture, followed by UV light exposure resulted in an increase in selfhealing properties of the mixtures prepared with PG 67-22 binder. Semi-Circular Bending (SCB) test results showed that the incorporation of self-healing polymer and $48 \mathrm{~h}$ of UV light exposure improved the cracking resistance. Loaded-Wheel Test (LWT) results showed that the self-healing polymer caused an increase in the rut depth of the samples prepared with an unmodified binder. However, the final rut depth was less than the acceptable rutting performance. Thermal-Stress Restrained Specimen Test (TSRST) test results showed that self-healing polymer improved the low temperature cracking performance of the mixtures.
\end{abstract}

\section{Introduction}

Asphalt binder has self-healing properties, which can be defined as the ability to restore its original properties. However, the self-healing rate of asphalt binder is extremely dependent on temperature and rest period. Cracks in asphalt pavements normally close at high temperature to reopen at low temperature. Using innovative self-healing techniques, fatigue cracking resistance of the pavement may be improved by accelerating the rate of crack healing. During the last decade, different self-healing techniques such as rejuvenators, encapsulated rejuvenators [1,2], induction heating [3], and nanoparticles [4] were examined to improve self-healing of asphalt mixtures. On the other hand, polymer additives have been used in the asphalt pavement industry to improve pavement's performance against fatigue cracking, thermal cracking, and rutting. Combining self-healing and polymer modification, a UV light activated self-healing polymer was examined in this study to improve the mechanical performance and selfhealing abilities of asphalt pavement containing recycled asphalt materials (RAS/RAP). The initiation and propagation of micro-cracks due to excessive loading and aging of asphalt binder leads to breakage of various bonds in the chemical structure of self-healing polymer, producing free radicals. The unstable free radicals would recombine through exposure to UV light, seal the microcracks, and stop crack propagation $[5,6]$.

\section{Objectives and scope}

This study has two main objectives: (1) to examine the effect of light-activated self-healing polymers on the self-healing abilities of asphalt mixtures, and (2) to evaluate the effects of light-activated self-healing polymers on mechanical properties of asphalt mixtures.

\section{Experimental program}

\subsection{Test materials}

Samples were prepared using two types of asphalt binders, PG 67-22 and PG 70-22M, with or without recycled asphalt materials (5\% RAS, and 20\% RAP), and with or without self-healing polymers. The RAS used was a Post-Consumer Waste Shingle (PCWS) with a $20 \%$ binder content, while the RAP used had a $5 \%$ binder content. The aggregate blend consisted of $5 / 8$ " gravel, 1/2" gravel, coarse sand and fine sand to satisfy the mix design for a $12.5-\mathrm{mm}$ Nominal Maximum Aggregate Size (NAMS) asphalt mixture. The Superpave asphalt mixtures were prepared in accordance with

\footnotetext{
* Corresponding author: marwa@1su.edu
} 
AASHTO R35-09, AASHTO M 323-07 and Section 502 of the 2006 Louisiana Standard Specifications for Roads and Bridges while using a Level 2 design. Ten asphalt mixtures were prepared and tested to evaluate the effects of self-healing polymer on the properties of the mixture (Error! Reference source not found.).

Table 1. Asphalt mixtures compositions.

\begin{tabular}{|c|c|c|c|}
\hline Binder Blend & $\begin{array}{c}\text { Binder } \\
\text { Type }\end{array}$ & $\begin{array}{c}\text { Recycled } \\
\text { Asphalt } \\
\text { Material }\end{array}$ & $\begin{array}{c}\text { Self-Healing } \\
\text { Polymer }\end{array}$ \\
\hline 67CO & PG 67-22 & 0 & 0 \\
\hline 67-5RAS & PG 67-22 & $5 \%$ RAS & 0 \\
\hline 67-5RAS-5P & PG 67-22 & $5 \%$ RAS & $5 \%$ \\
\hline 67-20RAP & PG 67-22 & 20\% RAP & 0 \\
\hline 67-20RAP-5P & PG 67-22 & 20\% RAP & $5 \%$ \\
\hline 70CO & PG 70-22 M & 0 & 0 \\
\hline 70-5RAS & PG 70-22 M & $5 \%$ RAS & 0 \\
\hline 70-5RAS-5P & PG 70-22 M & $5 \%$ RAS & $5 \%$ \\
\hline 70-20RAP & PG 70-22 M & 20\% RAP & 0 \\
\hline 70-20RAP-5P & PG 70-22 M & 20\% RAP & $5 \%$ \\
\hline
\end{tabular}

More information on the self-healing polymer test material, production process, chemical structure, and characterization were presented in a previous study. Moreover, in this study, the authors conducted Superpave PG grading and MSCR on binder blends prepared with PG $67-22$, with or without RAS (5\% by the weight of asphalt binder) and self-healing polymer $(1 \%, 3 \%$, and $5 \%$ by the weight of asphalt binder). Three different durations of UV exposure (1h, 24h, and 48h) was used. Results demonstrated that blends containing $5 \%$ self-healing polymer and exposed to UV light for $48 \mathrm{~h}$ showed the most significant improvements. As a result, $5 \%$ self-healing polymer and $48 \mathrm{~h}$ of UV exposure were selected for further testing [6].

\subsection{Effect of self-healing polymer on self- healing properties of asphalt mixture}

Crack healing rate of the mixtures prepared with and without self-healing polymer and recycled asphalt materials was examined using the Strain Energy Recovery Ratio (SERR). For sample preparation, rectangular beams of $40 \mathrm{~mm} \times 40 \mathrm{~mm} \times 160 \mathrm{~mm}$ dimensions were cut from compacted slab specimens. A three-point bending setup was used to apply a monotonic load at the midpoint of the beam in a strain-controlled mode $(0.25 \mathrm{~mm} / \mathrm{min})$ until cracks were induced at the bottom of the rectangular beams. Cracked samples were exposed to two different curing conditions; room temperature $\left(25 \pm 1^{\circ} \mathrm{C}\right)$ curing condition, and high temperature $\left(50 \pm 1^{\circ} \mathrm{C}\right)$ for samples without self-healing polymer and UV light exposure for samples containing self-healing polymer. The Strain Energy of samples was defined as the area under the load-deflection curve until failure (peak load). This value was measured for the undamaged samples (initial strain energy), and the healed samples after 6 days of curing (healed strain energy). Finally, the SERR was calculated as the ratio of healed strain divided by initial strain (equation 1).

$$
S E R R=\frac{\text { Healed Strain Energy }}{\text { Initial Strain Energy }}
$$

\subsection{Effects of light-activated self-healing polymer on the properties of HMA}

Laboratory performance of asphalt mixtures was tested using the SCB test, LWT test, and TSRST. SCB was conducted according to ASTM D 8044 to evaluate the cracking resistance of the prepared mixtures at intermediate temperature. For a better understanding of the polymer's effect on the cracking performance of the mixture, SCB was repeated with and without $48 \mathrm{~h}$ of UV light exposure. The resistance to permanent deformation of the mixtures was evaluated using the LWT according to AASHTO T 324. TSRST (AASHTO TP 10-93) was used to evaluate the mix resistance to fracture at low temperature.

\subsection{Statistical analysis}

A statistical analysis was conducted to determine whether differences in the performance of asphalt mixtures were significant. ANOVA and Tukey test were conducted to identify significant differences in the results. The analysis obtained from JMP software provided a grouping of the results using letters $(\mathrm{A}, \mathrm{B}, \mathrm{C}$, $\mathrm{D}$, and so forth). The letter A presented the highest mean, followed by the subsequent letters.

\section{Results and analysis}

\subsection{Effect of self-healing polymer on self- healing properties of asphalt mixture}

Results from SERR measurements of the PG 67-22 mixtures are presented in Error! Reference source not found.a. For samples conditioned at room temperature, the recovery ratio of the control mixture was improved from $38 \%$ to $72 \%$ due to addition of self-healing polymer. Furthermore, when recycled materials $(5 \%$ RAS and 20\% RAP) were added to the mix, a decrease in SERR was observed from $38 \%$ to $22 \%$ and $15 \%$, respectively. This decrease may relate to the effect of the aged and brittle binder in recycled materials. The selfhealing polymer incorporation to mixtures containing recycled asphalt material was able to partially recover the original SERR of the asphalt mixtures. Based on the results presented in Figure 1c and the statistical analysis performed, 67-5P mixture containing 5\% self-healing polymer presented the best recovery with an SERR value of $72 \%$, while $67-20$ RAP mixture containing $20 \%$ RAP showed the worst recovery ratio.

Error! Reference source not found.b presents the SERR results for samples exposed to high temperature/UV curing condition. Similar to results obtained for samples cured at room temperature, the maximum SERR (an SERR of 84\%) was measured for the mixture containing $5 \%$ self-healing polymer, while the control mixture shows an SERR of $52 \%$. The addition of 5\% RAS led to a statistically insignificant 
increase in the measured SERR of the sample. In contrast, the addition of $20 \%$ RAP decreased the measured SERR to a value of only $16 \%$. However, this decrease was partially recovered by the application of self-healing polymer and exposing samples to UV light for $48 \mathrm{~h}$. Finally, a statistical analysis conducted for curing condition comparison (Error! Reference source not found.c), showed that the improvement caused by the change of curing condition from room temperature to high temperature/UV exposure was not significant.

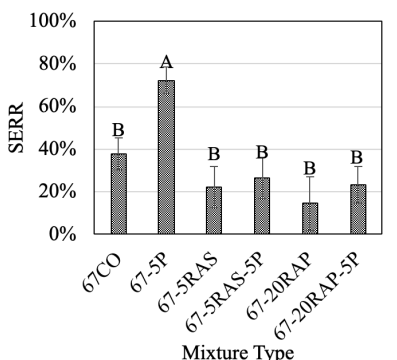

(a)

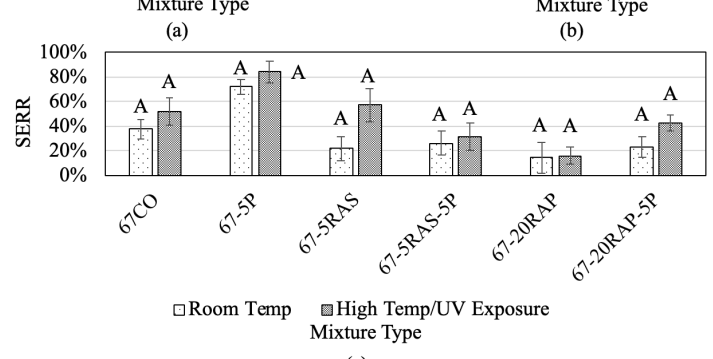

(c)

Fig.1. SERR results for PG 67-22 mixtures (a) room temperature curing, (b) high temperature/UV exposure curing, and (c) effect of curing condition.

Results from SERR measurements for the samples prepared using the PG 70-22M binder are presented in Error! Reference source not found. In this case, on the contrary of PG 67-22 mixtures, the addition of 5\% self-healing polymer resulted in a decrease in the calculated SERR. The application of recycled asphalt materials (5\% RAS and 20\% RAP) also led to a decrease in recovery ratio of the samples. The addition of 5\% selfhealing polymer in the mixture containing 5\% RAS increased the measured SERR from $9 \%$ to $34 \%$ at room temperature, and from $37 \%$ to $44 \%$ at high temperature/UV exposure. The same trend was observed for the mixture containing 20\% RAP. In conclusion, selfhealing polymer application followed by UV light exposure was able to improve crack recovery of the asphalt mixtures containing recycled asphalt materials; however, it did not completely recover the SERR of the control mix (70CO). Based on the statistical analysis performed (Error! Reference source not found.c), the high-temperature curing condition caused a significant improvement in healing recovery, while UV exposure curing condition did not have a significant effect.

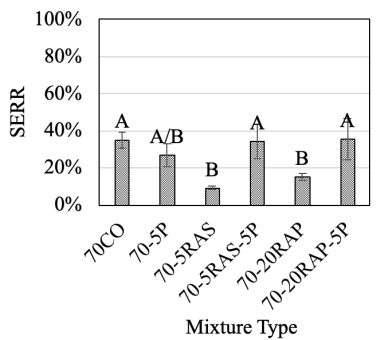

(a)

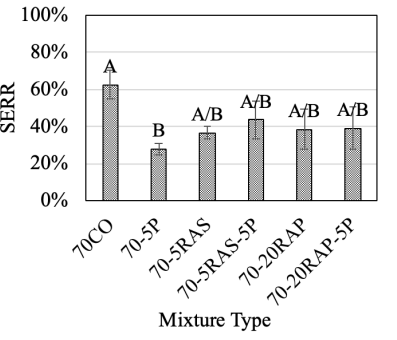

m(b)

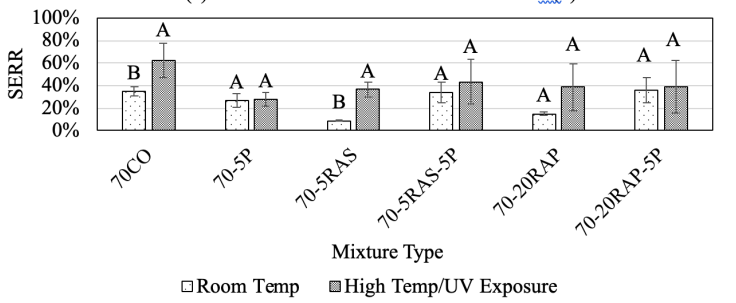

(c)
Fig.2. SERR results for $\mathrm{PG} 70-22 \mathrm{M}$ mixtures (a) room temperature curing, (b) high temperature/UV exposure curing, and (c) effect of curing condition.

\subsection{Effect of self-healing polymer on the mechanical properties of asphalt mixture}

\subsubsection{Semi-circular bending (SCB) test results}

The measured critical strain energy release rate $(\mathrm{Jc})$ of the mixtures evaluated in this study is presented in Error! Reference source not found.. For mixtures prepared with the unmodified binder (PG 67-22), the measured Jc value increased from $0.35 \mathrm{~kJ} / \mathrm{m}^{2}$ for the control mixture (67CO) to 0.38 , and $0.39 \mathrm{~kJ} / \mathrm{m} 2$ due to addition of $5 \%$ RAS (67-5RAS), and $20 \%$ RAP (6720RAP), respectively. The improvement in cracking resistance caused by the addition of recycled materials may be due to the polymers present in the recycled materials (RAS and RAP). However, all these values were less than the Louisiana's specification for Jc value of $0.5 \mathrm{~kJ} / \mathrm{m}^{2}$. Adding $5 \%$ self-healing polymer to the mixtures containing recycled asphalt materials (5\% RAS and $20 \%$ RAP) led to an increase in the measured Jc. However, based on the statistical analysis performed, this increase was only significant for mixtures with $20 \%$ RAP. Additionally, exposing samples to UV light for 48 hours led to an increase in the measured Jc value. For the mixtures prepared with polymer modified binder, when recycled asphalt materials were used, cracking performance was negatively affected. In this case, the Jc value of the control mix (70CO) decreased from 0.64 $\mathrm{kJ} / \mathrm{m}^{2}$ to 0.45 , and $0.62 \mathrm{~kJ} / \mathrm{m}^{2}$ for the cases of $5 \%$ RAS (70-5RAS), and 20\%RAP (70-20RAP). For mixtures containing $5 \%$ RAS, a Jc values of $0.72 \mathrm{~kJ} / \mathrm{m}^{2}$ demonstrated improved cracking performance through the use of 5\% self-healing polymer and $48 \mathrm{~h}$ of UV light. Yet, for a mixture containing 20\% RAP (67-20RAP-5P), the application of $5 \%$ self-healing polymer caused a decrease of the $\mathrm{Jc}$ value $\left(0.48 \mathrm{~kJ} / \mathrm{m}^{2}\right)$. 


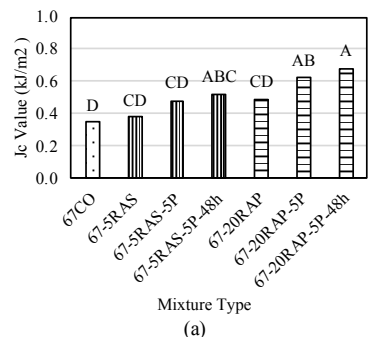

(a)

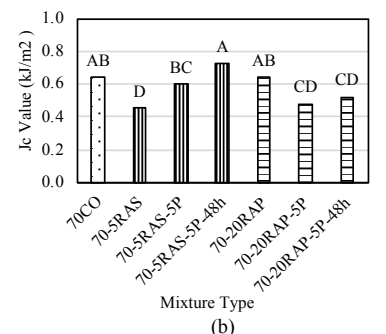

Fig.3. SCB results for (a) PG 67-22 mixtures, (b) PG 70-22M mixtures

\subsubsection{Loaded wheel tracking (LWT) test results}

Error! Reference source not found. presents the measured rut depths for the different asphalt mixtures. For the unmodified mixtures prepared with PG 67-22, the control mix (67CO) had the largest rut depth of 8.2 $\mathrm{mm}$ due to its low stiffness. However, when recycled asphalt materials ( $5 \%$ RAS, and $20 \%$ RAP) were added to the mix, the rut depth decreased to 3.2 , and 2.3, respectively. The use of 5\% self-healing polymer caused an increase in the measured rut depth for 67-5RAS-5P and 67-20RAP-5P mixtures; however, the measured rut depth was less than $6 \mathrm{~mm}$ and therefore below the failure criterion of Louisiana. For the PG 70-22M mixtures, the binder ( $\mathrm{PG} 70-22 \mathrm{M}$ ) is a polymer modified (SBS polymer) binder, which is known to improve the rutting performance at high temperature. Thus, the control mixture prepared with PG 70-22M binder (70CO) had a lower rut depth compared to the mixture prepared with PG 67-22 (67CO). The addition of recycled materials (5\% RAS, and 20\%RAP) decreased the measured rut depth as compared to the control mixtures with a rut depth of 2.5 , and $1.9 \mathrm{~mm}$, respectively. The addition of $5 \%$ self-healing polymer in to mixtures containing recycled asphalt materials (5\% RAS, and 20\%RAP) led to slight changes in the measured rut depth, however, changes were not statistically significant.

\subsubsection{Thermal-stress restrained specimen test (TSRST) results}

TSRST test results are presented in Error! Reference source not found. For mixtures prepared with PG 67-22 asphalt binder, the addition of 5\% RAS and 20\% RAP caused a slight decrease in the fracture temperature from $-16.8^{\circ} \mathrm{C}$ to and $-17.2^{\circ} \mathrm{C}$ and $-17.5^{\circ} \mathrm{C}$, respectively. This enhancement in low-temperature performance can be the outcome of the high polymer content in the RAP and RAS binders. The addition of 5\% self-healing polymer led to a further decrease in the fracture temperature, however, it was statistically insignificant. In conclusion, the use of RAP and RAS in this study did not appear to affect the fracture resistance of the mix at low temperature. When 5\% RAS and 20\% RAP were added to the mixtures prepared with $\mathrm{PG} 70-22 \mathrm{M}$, an increase and a decrease in fracture temperature was observed. Self-healing polymer caused a decrease in the measured fracture temperature for 70-5RAS-5P and 70-20RAP-5P.

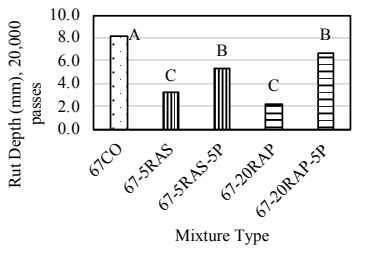

(a)

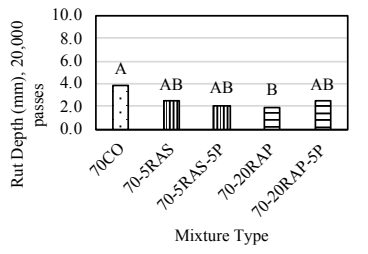

(b)
Fig.4. LWT results for (a) PG 67-22 mixtures, (b) PG 70-22M mixtures.
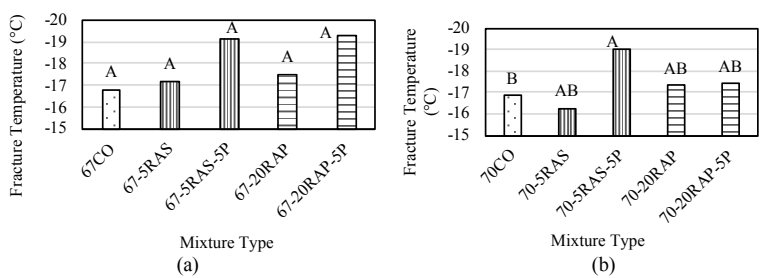

Fig.5. TSRST results for (a) PG 67-22 mixtures, (b) PG 7022M mixtures.

\section{Summary and conclusions}

The present study evaluated the effects of the addition of recycled asphalt materials (5\% RAS, and 20\%RAP) and a light-activated self-healing polymer $(5 \%)$ on the mechanical performance of asphalt mixtures. Using a set of laboratory tests, self-healing properties of asphalt mixtures were studied. Furthermore, the performance of asphalt mixtures against distresses such as permanent deformation, low temperature cracking and intermediate temperature cracking was characterized through LWT, TSRST, and SCB tests. Based on the results obtained from the experimental program, the following conclusions could be drawn:

- The use of recycled asphalt material in mixtures prepared with an unmodified binder negatively affected the crack healing recovery at room temperature. The addition of RAS to the mixtures also deteriorated the self-healing properties of $\mathrm{PG}$ 70-22M mixture, while in some cases, RAP addition resulted in improved properties. Self-healing abilities of the asphalt mixtures were improved by increasing the curing condition temperature from room temperature $\left(25^{\circ} \mathrm{C}\right)$ to high temperature $\left(50^{\circ} \mathrm{C}\right)$, which confirms the temperature dependency of the self-healing abilities of the asphalt mixtures. The addition of $5 \%$ Self-healing polymer to the control mixture, followed by UV light exposure for $48 \mathrm{~h}$ led to an increase in self-healing abilities of the mixtures prepared with unmodified binder. The highest crack healing recovery ratio (84\%) was observed for asphalt mixtures prepared with 5\% self-healing polymer, no recycled materials, and exposed to UV light for $48 \mathrm{~h}$. In the contrary, the application of self-healing polymer in PG 70-22M mixture resulted in a decrease in crack healing efficiency, which can be due to unwanted interactions of polyurethane with SBS in the binder. 
- $\quad$ SCB test results showed that when recycled asphalt materials were used, the cracking resistance of PG 67-22 mixtures were improved possibly due to the polymer content in the recycled asphalt materials sources. On the contrary, for mixtures prepared with PG 70-22M asphalt binder, recycled asphalt materials incorporation resulted in a decrease in the measured $\mathrm{J}_{\mathrm{c}}$ value from SCB test. The use of $5 \%$ self-healing polymer in the asphalt mixtures led to an improvement in the cracking resistance of the mixture, followed by a further increase through exposure to UV light.

- LWT test results stated that addition of recycled asphalt materials improved the rutting resistance of mixtures. Furthermore, the incorporation of the selfhealing polymer increased the rut depth of the mixtures prepared with an unmodified binder. However, except the $67 \mathrm{CO}$ mixture, all the final rut depth was less than allowed rut depth of $6 \mathrm{~mm}$.

- TSRST test results showed that the addition of recycled asphalt materials (5\% RAS and 20\% RAP) separately caused a decrease in the fracture temperature of the mixtures. A further decrease was observed through the application of self-healing polymer. However, differences were statistically insignificant.

This study is part of the project 17BSLU02 funded by the Transportation Consortium of South-Central States (Tran-SET) UTC and LTRC. The authors would also like to acknowledge the laboratory support from the Louisiana Transportation Research Center (LTRC) and the TGA work in the frame of the USDA S-1041 program.

\section{References}

1. Aguirre, M.A., Hassan, M.M., Shirzad, S., Daly, W.H., and Mohammad, L.N. (2016). Microencapsulation of asphalt rejuvenators using melamine-formaldehyde. Construction and Building Materials, Elsevier Ltd, 114, 29-39.

2. Shirzad, S., Hassan, M.M., Aguirre, M.A., Mohammad, L.N., Cooper, S., and Negulescu, I.I. (2017). Microencapsulated Sunflower Oil for Rejuvenation and Healing of Asphalt Mixtures. Journal of Materials in Civil Engineering, 29(9), 04017147.

3. García, Á.', Schlangen, E., Van De Ven, M., and Van Vliet, D. (2011). Induction heating of mastic containing conductive fibers and fillers. Materials and Structures/Materiaux et Constructions, 44(2), 499-508.

4. Hasaninia, M., and Haddadi, F. (2017). The characteristics of hot mixed asphalt modified by nanosilica. Petroleum Science and Technology, 35(4), 351-359.

5. Ghosh, B., Chellappan, K.V., and Urban, M.W. (2011). Self-healing inside a scratch of oxetanesubstituted chitosan-polyurethane (OXE-CHI-PUR) networks. Journal of Materials Chemistry, 21(38), 14473-14486.

6. Shirzad, S., Hassan, M.M., Aguirre, M.A., Cooper, S., and Negulescu, I.I. (2018). Effects of LightActivated Self-Healing Polymers on the Rheological Behaviors of Asphalt Binder Containing Recycled Asphalt Shingles. Transportation Research Record. 